\title{
Development and Application of a MHz Frame Rate Digital Particle Image Velocimetry System
}

\author{
Mark P. Wernet ${ }^{*}$ \\ NASA Glenn Research Center \\ Cleveland, OH 44135
}

\author{
Anthony B. Opalski ${ }^{\dagger}$ \\ QSS Group, Inc. \\ Cleveland, OH 44135
}

\begin{abstract}
The ability to capture the unsteady dynamics of high speed compressible flows requires the use of ultra fast imaging diagnostics. Application of particulate based scattering techniques in high speed flows requires the use of high power, nanosecond duration, pulsed illumination sources such as Nd:YAG lasers in order to record high quality images. Furthermore, the temporal bandwidth of the measurements must be at least several hundreds of $\mathrm{kHz}$ while the spatial scale must be at least on the order of the large-scale structures present in the flow. High spatial and temporal bandwidth flow field measurements are not simultaneously available with high accuracy due to the combined constraints imposed by high sensitivity digital image sensors and limited pulse repetition rates of solid-state lasers, respectively. In this work we describe the development of a $\mathrm{MHz}$ repetition rate, high resolution, quantitative flow measurement system. The imaging system is comprised of a $\mathrm{MHz}$ repetition rate pulse-burst mode laser built in-house and a commercial $\mathrm{MHz}$ framing rate charged coupled device (CCD) camera. The laser is capable of producing a wide range of inter-pulse spacings (0.5 to 20 $\mu \mathrm{s})$ with individual pulse energies exceeding $75 \mathrm{~mJ}$ at $532 \mathrm{~nm}$. The camera, having a single optical input, is comprised of an internal image splitter unit and four individual intensified, frame-straddling, 1280 x 1024 pixel, CCD camera modules. The combined laser and camera system provides imaging frame rates ranging from $2 \mathrm{MHz}$ down to $50 \mathrm{kHz}$. Results from the application of the $\mathrm{MHz}$ rate imaging systems to obtain Digital Particle Image Velocimetry (DPIV) data from a supersonic nozzle flow will be presented.
\end{abstract}

\begin{abstract}
A. Introduction
The assessment and validation of advanced combined cycle propulsion system components requires detailed mapping of flow field features during unsteady transient operation. Planar optical diagnostic techniques lack the temporal resolution to provide the requisite measurements. In addition to the requirement of high temporal resolution, the optical diagnostic must be compatible with the confined spaces/flow passages in these prototype propulsion systems. For practical flow fields of interest (compressible/turbulent/high Reynolds number), the temporal bandwidth must be at least several 100's of kHz, while the spatial scale must be at least on the order of the large-scale structures present in the flow. High spatial and temporal bandwidth flow field measurements are not simultaneously achievable with high accuracy due to the combined constraints imposed by high sensitivity digital image sensors and the limited pulse repetition rate of solid-state lasers, respectively. Orders of magnitude increase in the capability of monitoring unsteady flow phenomena is necessary for the complex flow field changes that will occur during the rapid cycle mode transitions in advanced propulsion systems.
\end{abstract}

For particulate based optical diagnostic systems, typically short duration, high energy output pulses from Nd:YAG lasers are required in order to provide sufficient flow field illumination. The pulse repetition rate of commercially available Nd:YAG laser systems has traditionally been limited to the range of 10 to 30 pulses/second. Newer Nd:YAG laser systems are becoming available that operate in the $\mathrm{kHz}$ regime, with pulse energies approaching $10 \mathrm{~mJ}$. Copper vapor lasers have been available in the $10 \mathrm{kHz}$ operation range for many years, [Wieneke and Reckers, 2000]. In order to study the transient phenomenon occurring in advanced propulsion systems, we require pulsed laser systems that operate at even higher repetition rates, in the $\mathrm{MHz}$ regime, with pulse energies exceeding even those available from the current $10 \mathrm{kHz}$ laser

\footnotetext{
* Senior Research Engineer, Associate Fellow, AIAA

${ }^{\dagger}$ Research Engineer, Member, AIAA
} 
systems. Burst mode, MHz repetition rate Nd:YAG laser systems having pulse energies $\approx 10 \mathrm{~mJ}$ have been developed and demonstrated by Lempert, et al., 1996 and 1997.

Typical commercially available, high sensitivity CCD cameras are similarly constrained to low temporal resolution operation $(30 \mathrm{~Hz})$. These rates are too slow, by many orders of magnitude, to track dynamic changes in high speed flows. Frame-straddling Particle Imaging Velocimetry (PIV) type cameras can be used to acquire image frame pairs, but the frame pairs are still only acquired at low temporal resolution $(15 \mathrm{~Hz})$. Cinemagraphic PIV measurements have been obtained at frame rates up to $8 \mathrm{kHz}$ using film based cameras, but require tedious digitization of the film and frame-to-frame registration, [Upatnieks, et al., 2002]. High temporal resolution camera systems capable of recording image data sequences at $\mathrm{MHz}$ rates are divided into two types: 1) single sensor, low spatial resolution systems; and 2) multi-sensor, high spatial resolution systems. In the work of Thurow, et al., 2004, a MHz laser system was coupled with a single, low resolution, high framing rate CCD to provide Planar Doppler Velocimetry (PDV) based single-component velocity measurements of a high speed turbulent nozzle flow. High resolution, multi-sensor camera systems have been used in low velocity flows to obtain DPIV measurements at a $10 \mathrm{kHz}$ framing rate, [Wieneke and Reckers, 2000].

In this work we describe a quantitative optical diagnostic system that employs a burst mode $\mathrm{MHz}$ laser system, which is capable of producing individual pulse energies on the order of $75 \mathrm{~mJ}$ at $532 \mathrm{~nm}$ combined with a multi-sensor, high spatial resolution camera system also capable of $\mathrm{MHz}$ rate operation. The combined $\mathrm{MHz}$ rate imaging system enables for the first time, quantitative flow measurements via MHz rate DPIV.

\section{B. MHz Repetition Rate Pulse Burst Laser}

In 1999, the NASA Glenn Research Center took delivery of a MHz rate, pulse burst mode laser system developed under an Small Business Innovative Research (SBIR) contract. The SBIR developed laser system was designed to use a pulsed laser diode as the seed laser, which was anticipated to remove the pedestal buildup in "slicer" based $\mathrm{MHz}$ laser systems. Difficulties encountered in implementing this approach led to poor system performance and inflexible inter-pulse spacings. The core elements of the SBIR laser system were used along with new components to build a MHz rate, burst mode laser system based on the design previously demonstrated by Lempert, et al., 1997.

The layout of the MHz rate, burst mode laser is shown schematically in figure 1. The $1064 \mathrm{~nm}$ output from a 300mW continuous wave (cw), diode pumped Nd:YAG seed laser (which serves as the primary oscillator) is pre-amplified in a flashlamp-pumped, pulsed amplifier (AMP 1) configured in a double-pass mode of operation. The resulting laser pulse, of approximately $150 \mu$ s duration, is formed into a "burst" train of pulses using a custom, dual Pockels cell beam "slicer". The resulting pulse train can have a variable number of pulses, ranging between 1 and 99, with individual pulse durations ranging between 2 and 10,000 ns and interpulse spacings ranging between 500 and 20,000 ns. The number, duration and spacing of the pulses in the "burst" train is quite flexible and ultimately is limited only by the period ( 150 $\mu s)$ during which the flashlamp-pumped amplifiers have sufficient gain. The repetition rate of the burst sequence can be as high as 10 $\mathrm{Hz}$, which is limited by the thermal loading of the amplifiers and optics. A typical burst train for the work described here consists of eight, $10 \mathrm{~ns}$ duration pulses, separated by 2 to $8 \mu \mathrm{s}$, and were generated at a conservative repetition rate of $5 \mathrm{~Hz}$.

The output pulse train from the slicer is further amplified in a series of five flashlamp-pumped amplifiers with increasing rod diameters of $4.0 \mathrm{~mm}$ (Amps 1\&2), $6.35 \mathrm{~mm}$ (Amps 3\&4), $9 \mathrm{~mm}$ (Amp 5) and $12.7 \mathrm{~mm}$ (Amp 6). Three of the amplifier stages (AMPS 2, 3 and 4) are operated in a double-pass mode and two are configured for single-pass (AMPS 5 and 6) operation. Following this amplification chain, the output is converted to the second-harmonic (532 nm) with a $12 \mathrm{x}$ 12 x 8 mm Type II KTP Second Harmonic Generation (SHG) crystal operating at room temperature.

A novel feature of the laser is the incorporation of a Phase-Conjugate Mirror (PCM) into the burst mode laser amplification chain (after AMP 3), Lempert et al., 2004. As can be seen in figure 1, the pulse train is focused into the PCM after the third amplifier (with a $75 \mathrm{~mm}$ focal length lens) and is coupled to the final three amplifiers using a quarter wave plate/thin film polarizer combination. The PCM is comprised of a $25.4 \mathrm{~mm}$ dia. by $101.6 \mathrm{~mm}$ long optical cell, filled with a 1.28 index of refraction inert liquid (3M Flourinert FC-75).

The PCM serves two important purposes in the current laser system layout. The first is to remove a low-intensity "pedestal" superimposed upon the high-intensity pulses which make up the output burst train. The pedestal forms as a consequence of $\sim 0.1 \%$ leakage through the Pockels Cells when the units are in the normally off state. Since the slicer is operating (on) for only 10 ns out of $\sim 10 \mu \mathrm{s}$, this results in substantial integrated energy in the pedestal, which becomes preferentially amplified relative to the pulses in the later amplification stages. The PCM is able to remove this pedestal by operating under the principle of Stimulated Brillouin Scattering (SBS), which is a nonlinear optical phenomenon in which a 
coherent backscattered beam (at $180^{\circ}$ ) is produced if an incident input beam achieves a minimum intensity level (SBS threshold). By placing the PCM at the appropriate spot in the amplifier chain after AMP 3, it is possible to exceed the SBS threshold intensity for the desired pulses, yielding a reflected beam which is directed back into the amplification chain, while keeping the pedestal intensity well below threshold. The pedestal passes through the PCM into a beam dump and, hence, is removed from the optical chain.

The second purpose of the PCM is to minimize the growth of Amplified Spontaneous Emission (ASE) by providing a necessary isolation (break in the growth chain) between the first three and last three amplification stages. Similar to the pedestal problem described above, ASE occurs when the gain becomes sufficiently high that laser output occurs even in the absence of any input from the master oscillator (seed laser). The ASE increases exponentially from noise as the pulse train progresses downstream through the optical chain. If the intensity of the ASE component after the third amplifier is well below threshold, it also passes through the PCM and is removed from the amplification chain. The ASE build-up process then begins again with the spontaneous emission from the fourth amplifier.

Figure 2 shows the physical layout of all of the components in the laser system. All flashlamp pulse forming network (PFN) circuit electronics are contained within the single rack mount enclosure. Laser rod and flashlamp cooling is provided by an independently controlled $4 \mathrm{~kW}$, closed-loop heat exchanger. The optical components of the laser are contained on a 91×152×10 cm optical breadboard and encased within a removable enclosure.

The performance of the burst mode laser has been evaluated for a range of burst sequences and pulse energy levels. Average second-harmonic conversion efficiency has been shown to exceed 50\%, with all amplifiers operated at well below their maximum electrical input energy levels. Increased spacing between pulses has been shown to correlate to higher pulse energies. The spectral linewidth (FWHM) of the burst mode laser is anticipated to be of the order $50 \mathrm{MHz}$ for a typical 12 ns pulse, [Thurow, et al., 2004]. The laser system's narrow linewidth and excellent pulse-to-pulse spectral stability are both critical to its use in a Doppler Global Velocimetry (DGV) measurement application. Tunability of the cw seed laser frequency (1064.03 to $1064.24 \mathrm{~nm}$ ) has also been provided to facilitate implementation of the DGV technique.

Measured output pulse energies to date have exceeded $75 \mathrm{~mJ} / \mathrm{pulse}$ (8 pulse train, $10 \mathrm{~ns}$ pulses, $2 \mu$ separation) at $532 \mathrm{~nm}$. Excellent pulse energy uniformity has been observed for relatively low amplifier energy inputs. Figure 3 shows an oscilloscope trace of a moderate energy $532 \mathrm{~nm}$ burst train (10 pulses with an inter-pulse spacing of $2 \mu \mathrm{s}$ and an average pulse energy of less than $20 \mathrm{~mJ} / \mathrm{pulse}$ ). The top trace is the electrical pulse train sent to the pulse slicer unit and the bottom trace is the actual laser output recorded via a photodiode. Although modulation in the pulse train envelope can be expected to approach $\pm 50 \%$ of the mean value (at the nominal design amplifier energy settings), flexibility in specifying the number of pulses in the resultant train should readily provide a window of uniform pulses to be extracted for use in required applications. If higher output energies are required, the current single-pass mode of operation of AMP 5 can be modified to accommodate a dual-pass configuration. The use of high quality, short, stiff optical mounts provides a very stable optical system. No alignment of the laser system is required for day to day operation. The laser system was transported by truck to another NASA GRC facility, and after electric hookup, required only minor adjustment of 2 mirrors to restore optimal system alignment.

\section{High-Speed Camera}

Image data illuminated using the $\mathrm{MHz}$ rate, burst mode laser have been acquired using an HFSC-Pro camera, supplied by the Cooke Corporation. Having a single optical input, the HSFC-Pro camera system comprises an image splitter unit and four intensified, frame-straddling, CCD camera modules with fast switching Micro-Channel Plate image intensifiers and high resolution, 1280 x 1024 pixel, Peltier cooled, CCD image sensors. The layout of the internal camera components is shown in figure 4. Each module's CCD sensor has high bit depth (12 bits per pixel), high signal-to-noise-ratio (69 dB) and moderate quantum efficiency (30\%) at $532 \mathrm{~nm}$. The image splitter module ensures that the optical paths for all four cameras are matched and provides an optical efficiency of approximately 23\% to each CCD module. Four high speed fiber optic data links connect the system to a control PC, which can be externally triggered. The intensifiers, equipped with a fast decay P46 phosphor, enable inter-framing times down to $500 \mathrm{~ns}$, which allows a sequence of 8 full resolution image frames to be acquired in rapid succession.

Although the camera system was originally delivered with internal beamsplitting cubes and prisms which are lightpolarizing, arrangements have been made to replace these image splitter components with non-polarizing optics for compatibility with DGV applications. An investigation is also underway regarding the use of imaging fiber bundles to couple the scattered laser light to the camera system, which will facilitate the use of this otherwise large camera system in a confined space application. 


\section{Experimental Setup}

Initial plans to apply the $\mathrm{MHz}$ rate imaging system to a pulsejet flow were not successful due to facility commitment issues. Instead a small scale, $8.8 \mathrm{~mm}$ diameter, convergent-divergent Mach 1.3 nozzle was setup in the lab. In order to produce a non-stationary flow, two constant diameter $\left(2 \mathrm{~mm}\right.$ ) flow excitation jets were oriented at $55^{\circ}$ to the nozzle axis as shown in figure 5. Pneumatic actuators were used to switch the excitation jets at $10 \mathrm{~Hz}$. The jet core flow was seeded in the nozzle plenum section using olive oil and a 6-jet atomizer. Ambient flow seeding was provided by a second 6-jet atomizer also filled with olive oil. The nominal particle size obtained using these atomizers is $0.7 \mu \mathrm{m}$. Attempts were made to seed the excitation jet flow, but proved difficult due to the discontinuous operation mode.

The output from the $\mathrm{MHz}$ laser system was directed from above the nozzle axis centerline and formed into a downward propagating light sheet, terminating into a beam dump. The $\mathrm{MHz}$ rate camera system was oriented at $90^{\circ}$ to the jet axis and positioned $80 \mathrm{~cm}$ from the light sheet plane. The imaged area of the light sheet started $8 \mathrm{~mm}$ from the nozzle exit and covered a total area of $90 \times 75 \mathrm{~mm}$. The laser was configured to produce a 10 pulse train of which the most uniform amplitude central 8 pulses were used for image exposures. Since each CCD is capable of operating in frame-straddling mode, a pair of A/B image frames are obtained from each CCD module. The laser/camera system was operated such that laser pulse \#1 exposed CCD1 frame A, pulse\#2 exposed CCD1 frame B, pulse \#3 exposed CCD2 frame A, pulse \#4 exposed CCD2 frame B, ... pulse \#8 exposed CCD4 frame B. Two exposure sequence schemes were used in this work: uniform inter-pulse spacings and a short-long pulse spacing scheme, each of which are depicted in figure 6. In figure 6a, the short-long inter-pulse spacing exposure sequence and processing strategy is shown, where four velocity vector maps with long inter-vector map delay time delays are obtained. Figure 6b, illustrates a uniform inter-pulse spacing acquisition and processing strategy, where the 8 single exposure images are acquired and processed to obtain 7 velocity vector maps.

Since the camera head contains four independent sensors, we need to perform cross-CCD correlations in order to extract 7 velocity vector maps from 8 image frames. In order to perform these cross-CCD correlations, an image warping correction is required. In standard stereoscopic PIV processing, the preferable approach is to correlation process the raw, distorted CCD images and then perform the warping on the computed velocity vectors. Experience has shown that warping/interpolating the raw images leads to a loss of correlation, and a general degradation of the velocity vector maps. For the multiple camera head system used here, we are forced to perform image warping prior to cross-correlation PIV data processing in order to obtain our 7 velocity vector maps.

A multi-plane calibration, similar to that used in stereo PIV, was performed on the camera system, [Soloff et al., 1997]. The calibration was performed using a single plane calibration target that was traversed through five z-axis planes, which spanned the thickness of the laser light sheet. The coordinates of the calibration markers across all five planes were used to determine the calibration warping polynomial coefficients. CCD's 1 and 2 were treated as a stereo imaging pair and similarly CCD's 3 and 4 were treated as a stereo pair. The calibrations for each stereo CCD pair were scaled to a common magnification and fiducial point, yielding pixel registered images across all four modules. Figure 7 shows the results of warping the calibration grids for all four modules to a common grid. The resulting rms variation in the warped calibration marker locations is 0.25 pixels. The image intensities were interpolated in the warping process using a bicubic interpolation. Cross-correlation of the warped/interpolated PIV images yielded high quality velocity vector maps. Figure 8 shows a sample image obtained from the high framing rate camera system. Operating the image intensifiers at low gain yields high quality, low blooming PIV image data with clearly resolved particle images.

\section{E. Results and Discussion}

An ensemble average of 256 velocity vector maps is shown in figure 9a and 9b, for the no-pulse and pulsed excitation jet cases, respectively. The color contours represent the velocity vector magnitude and every other column of vectors have been omitted for clarity. The Mach 1.3 nozzle was operated at a plenum pressure ratio of 0.36, which should have produced a matched flow condition at the exit. Shock diamonds are readily apparent in the ensemble averaged velocity fields, indicating an under expanded flow condition. The laboratory air supply line may not have been able to supply the requisite mass flow in order to obtain a matched pressure condition at the nozzle exit. The maximum jet velocity measured was $410 \mathrm{~m} / \mathrm{s}$, whereas the expected velocity based on the isentropic flow equations was $447 \mathrm{~m} / \mathrm{s}$. The effect of the pulsed excitation jet is to squeeze the width and shorten the length of the jet potential core. It was also anticipated that the excitation jets would break up the jet core flow yielding large scale vertical structures, which could be recorded using the $\mathrm{MHz}$ imaging system. The true magnitude of the excitation jet flow is not resolvable in the ensemble average plot, however, from the single shot velocity vector maps the peak velocity in excitation jet flow was observed to be approximately Mach 0.5 . 
Uniformly spaced and non-equispaced time resolved image data were acquired with the MHz rate PIV system. A $2 \mu$ s equispaced sequence was acquired from the flow without pulsed excitation. The nominal laser pulse energy was $25 \mathrm{~mJ}$. The image data were warped to a common grid and cross-correlation processed. Only four out of the seven vector maps are shown in figure 10 (velocity maps $1,3,5$, and 7 out of the sequence) and the vertical extent of the vector map has been clipped to just the region of the jet core flow. The velocity vectors are drawn in black, the color contours represent the vector magnitude and the nozzle exit plane is located at $x=0 \mathrm{~mm}$. The cross-correlation processed vector maps shown here used 64x64 pixel subregions on 16 pixel centers, yielding interpolated velocity vector maps. Good quality velocity vector maps were also obtained using 32x32 pixel subregions on 16 pixel centers; however, the interpolated velocity vector maps have better uniformity and help the reader to better track the coherent structures in the fluid as they convect downstream. Coherent structures both in the shear layer and downstream of the potential core are observed convecting downstream in the time evolution sequence.

A non-equispaced image sequence was acquired from the pulsed excitation jet flow, where the inter-pulse time was $2 \mu$ and the time between vector maps was $8 \mu \mathrm{s}$. The pulse amplitude uniformity across the burst becomes more difficult to maintain for these non-equispaced pulse trains. Adjustment of the flashlamp delay times is required to obtain generally uniform pulse energies. The nominal pulse energy for this image sequence was $35 \mathrm{~mJ}$. Figure 11 shows the four velocity vector maps obtained from the pulsed excitation jet flow. Again the velocity vectors are drawn in black and the color contours represent the velocity magnitude. Poor flow seeding in the pulsed excitation jet yielded an ill defined excitation flow, although its extent and location are readily identified. The error in these velocity measurements varies depending on whether the correlations are performed inter- or intra-camera. For intra-camera correlations the error is nominally $1 \%$ of full scale, while for the inter-camera correlations, the full scale error is nominally $2 \%$, due to the image warping process. There are several features of note in this sequence. First, the sequence appears to have captured the excitation jet impact on the nozzle flow. In the third vector map in the sequence, the nozzle flow narrows significantly as the pulsed jet flow impinges on the nozzle core flow. The effect of the excitation jet is to pinch off a high speed packet of fluid, which appears to be one of the original shock diamonds in the under expanded nozzle flow. The high speed jet core flow is then observed to roll up into larger diameter vortex structures that continue to convect downstream with a noticeable speed reduction. Entrainment of the low speed ambient fluid is also observed at approximately $80 \mathrm{~mm}$ downstream from the nozzle exit.

\section{F. Conclusions}

A $\mathrm{MHz}$ repetition rate laser/camera system has been demonstrated to provide high temporal and high spatial resolution DPIV image data in a supersonic nozzle flow. The MHz repetition rate burst mode laser system was demonstrated to generate both equispaced pulse trains and non-equispaced pulse trains. In both cases relatively uniform pulse energies across the 8 pulse train were obtained. The high resolution, multi-CCD head camera system was easily synchronized with the laser pulses. A multi-plane calibration process was used to pixel register all four of the CCD sensors in the camera system. Using a bicubic interpolation scheme, the warped images could still be processed using PIV cross-correlation techniques to provide high quality velocity vector maps. DPIV image data were acquired from a lab scale supersonic nozzle flow under two different operating conditions. Disturbances in the nozzle flow under the influence of external excitation pulsed jets were resolved with the MHz DPIV system. Structures in the shear layer of the jet were easily tracked as they were convected downstream.

Future plans include the use of the $\mathrm{MHz}$ imaging system in a high speed transient pulsejet and/or pulse detonation engine flow, where the high temporal resolution features of the system can be exploited to resolve the features of the high speed pulsed flow. In order to more readily facilitate the transport of the $\mathrm{MHz}$ laser system to research test facilities, the entire 6 amplification stage layout is being migrated to a reduced size optical table.

\section{G. Acknowledgements}

The authors would like to thank Prof. Walter Lempert for his assistance in the component selection and design of the $\mathrm{MHz}$ laser system. The authors also recognize the critical input from both Brian Thurow and Naibo Jiang in the optimization of the burst mode laser system performance. 


\section{H. References}

Lempert, W. R., Wu, P. F., Zhang, B., Miles, R. B., Lowrance, J. L., Mastocola, V. J., Kosonocky, W. F., 1996, “Pulse Burst Laser System for High Speed Flow Diagnostics”, AIAA-96-0179, Presented

at the AIAA $34^{\text {th }}$ Aerospace Sciences Meeting and Exhibit, Reno, NV, January.

Lempert, W. R., Wu, P. F., Zhang, B., Miles, 1997, "Filtered Rayleigh Scattering Measurements Using a MHz Rate PulseBurst Laser System”, AIAA-97-0500, Presented at the AIAA 35 ${ }^{\text {th }}$ Aerospace Sciences Meeting and Exhibit, Reno, NV, January 6-9.

Lempert, W., Jiang, N., Blohm, M., Thurow, B., Samimy, M., Switzer, G. and Goss, L., 2004, “A Burst Mode OPO System for $\mathrm{MHz}$ Frame Rate PLIF Imaging Diagnostics,” AIAA-2004-0387, Presented at the 42nd AIAA Aerospace Sciences Meeting, Reno, NV, Jan 5-8.

Soloff, S. M., Adrian, R. J., Liu, Z-C., 1997, “Distortion Compensation for Generalized Stereoscopic Particle Image Velocimetry”, Meas. Sci. Technol., 8, pp. 1441-1454.

Thurow, B., Jiang, N., Lempert, W., Samimy, M., 2004, “MHz Rate Planar Doppler Velocimetry in Supersonic Jets”, AIAA-2004-0023, Presented at the 42 ${ }^{\text {st }}$ AIAA Aerospace Sciences Meeting, Reno, NV, January.

Upatnieks, A., Laberteaux, K.L., Ceccio, S.L., 2002, “A Kilohertz Frame Rate Cinemagraphic PIV System for LaboratoryScale Turbulent and Unsteady Flows,” Experiments in Fluids, Vol. 32, Issue 1, 87-98.

Wieneke, B. and Reckers, W., 2000, "High-Speed PIV Using High-Frequency Diode-Pumped Solid State Laser and MultiFrame CCD”, $9^{\text {th }}$ International Symposium on Flow Visualization, paper No. 439. 


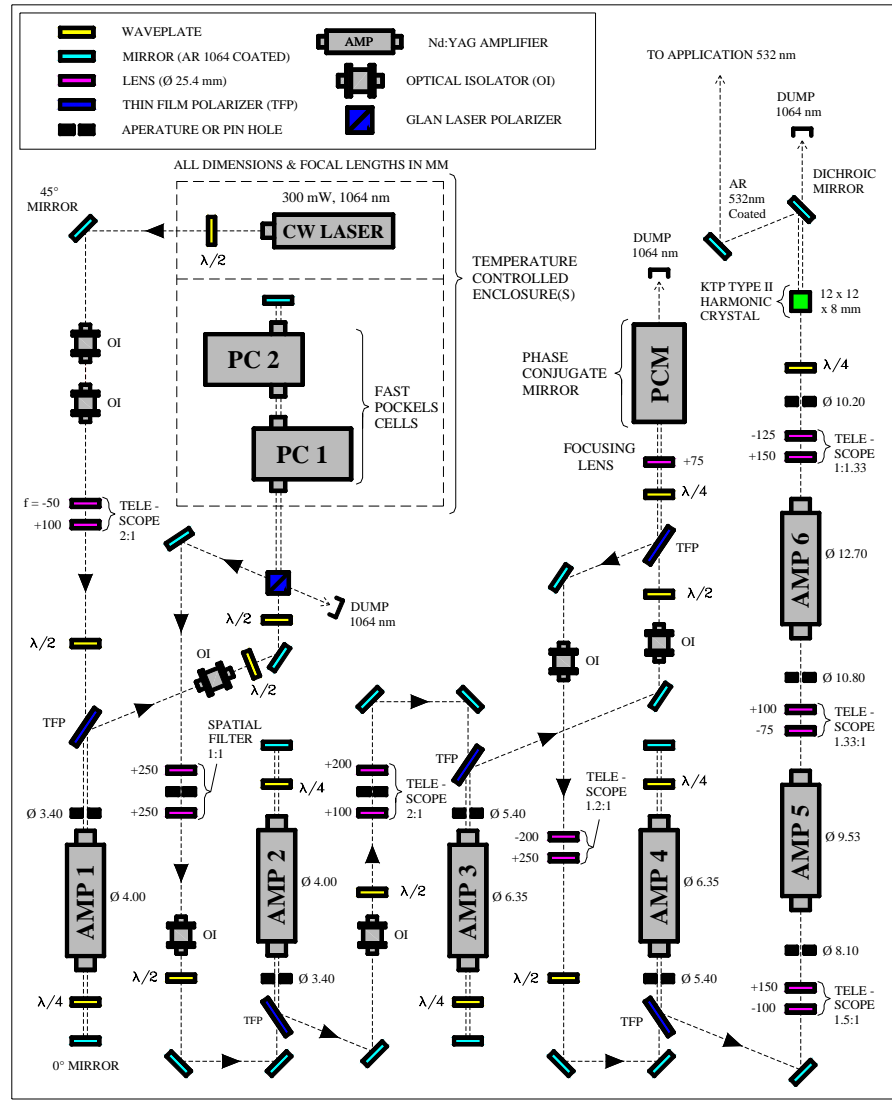

Figure 1: Schematic illustrating current configuration of $\mathrm{MHz}$ repetition rate pulse burst laser system.

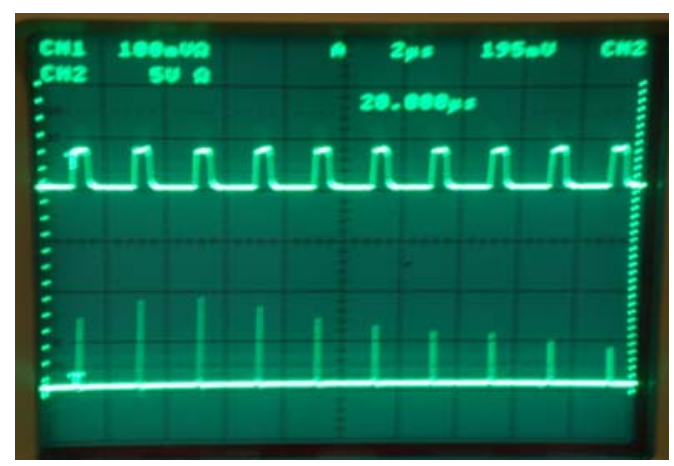

Figure 3: Oscilloscope trace of typical 10 pulse train illustrating envelope modulation. The top trace is the electric signal sent to the pulse slicer and the bottom trace is the actual laser output energy.

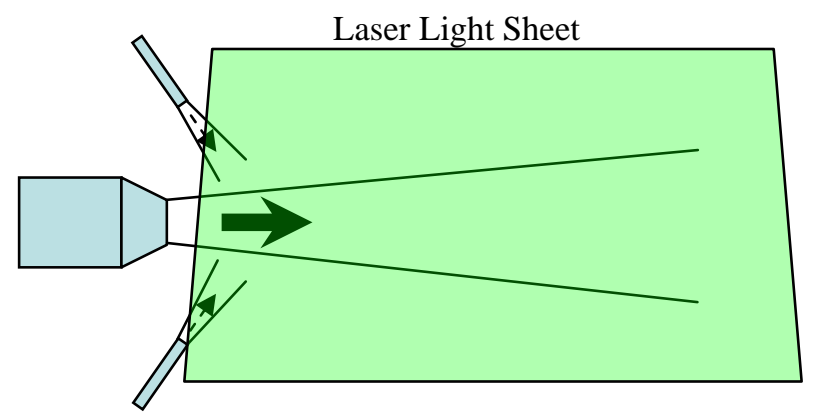

Figure 5: Schematic layout of nozzle with excitation jets oriented at 55 degrees to the jet axis. The laser light sheet is propagating in the downward direction.

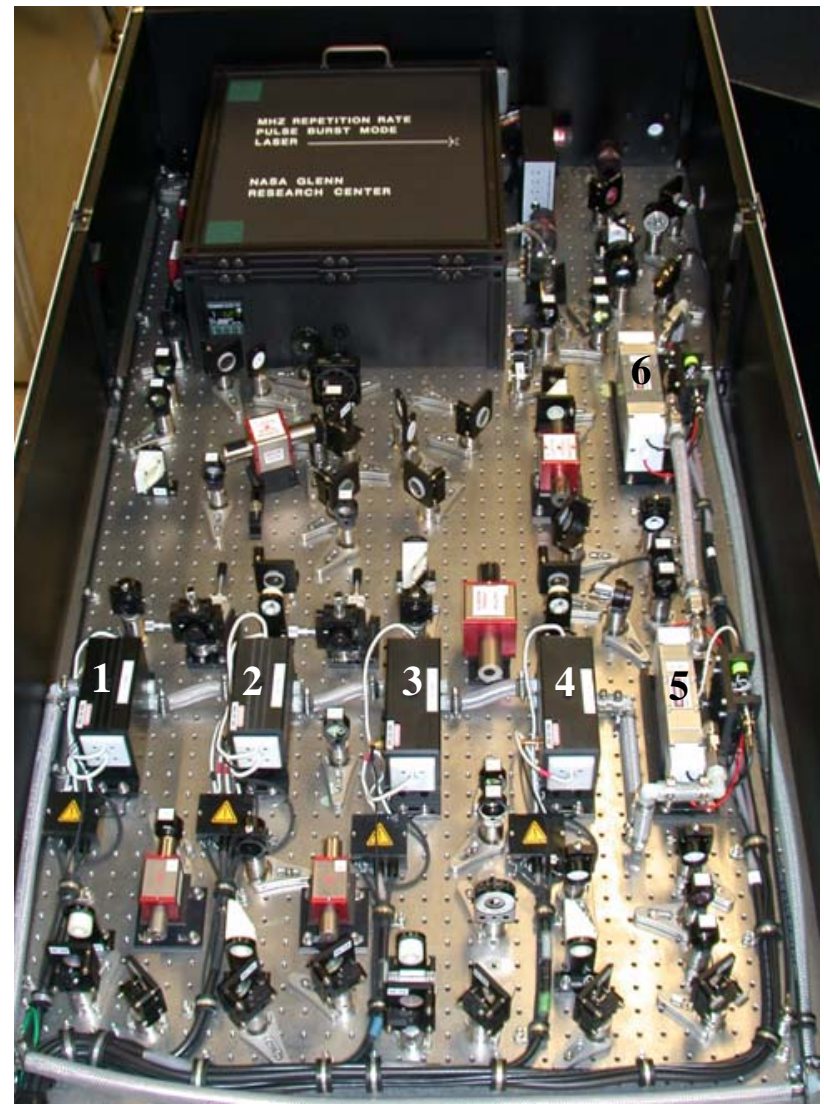

Figure 2: Photograph showing layout of pulse burst laser components on optical breadboard (enclosure cover removed).

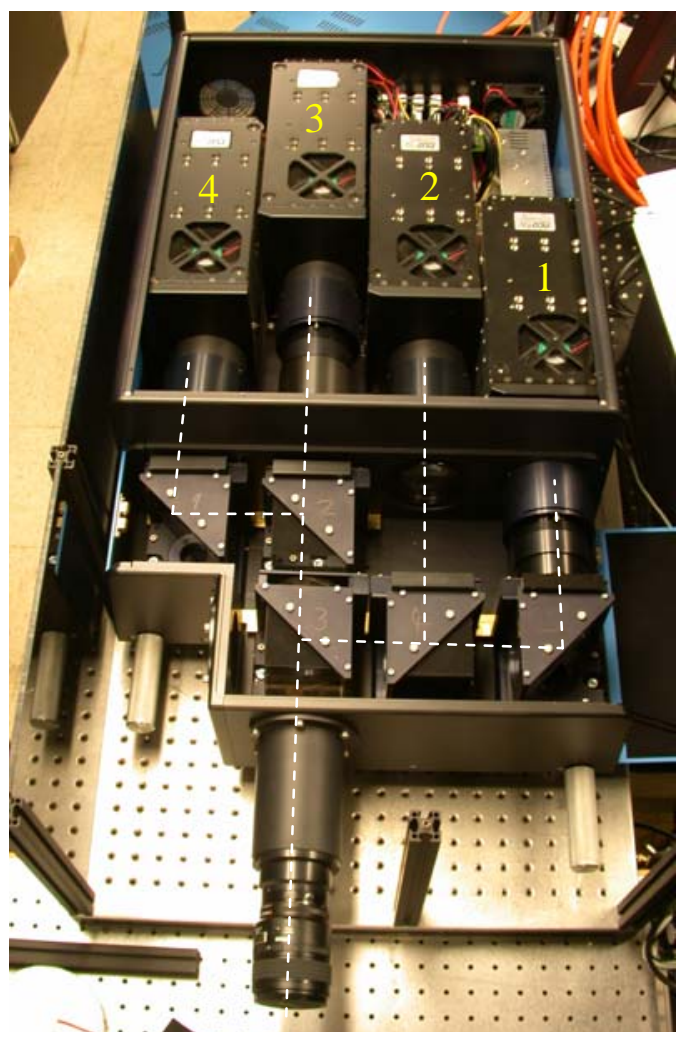

Figure 4: Layout of the high speed, high resolution camera system. The optical paths for the four separate cameras are delineated by the dashed lines. Light enters a single input objective and is split by a series of beam splitting cubes and prisms. 
a)

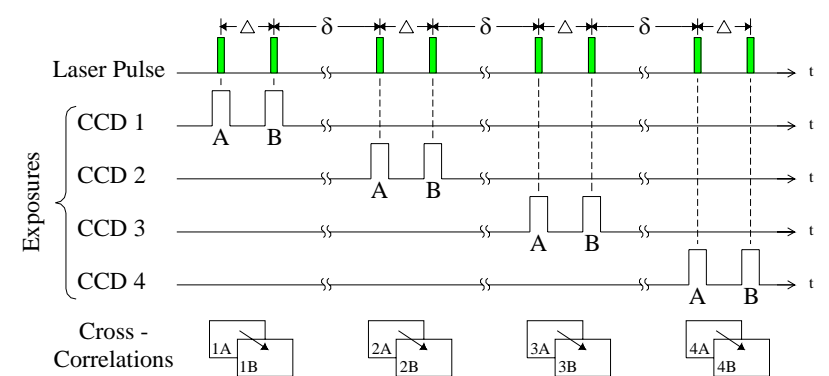

b)

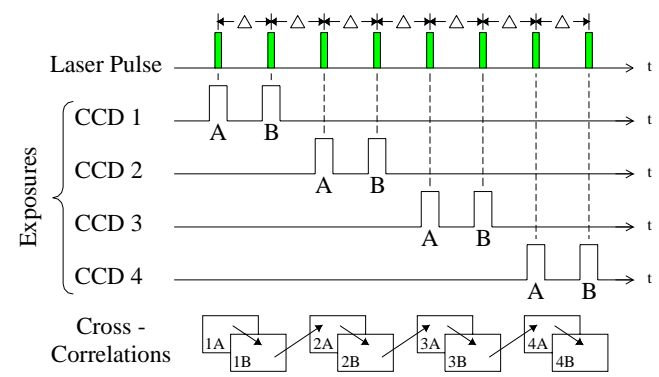

Figure 6: Two different image exposure sequence schemes: a) the scheme on the left shows a short inter-frame time followed by a long pause, where intra-camera frame-straddle images are cross-correlation processed; $b$ ) the scheme on the right shows equispaced pulses, where now both intra- and inter-camera images frames are cross-correlation processed to yield 7 velocity vector maps.

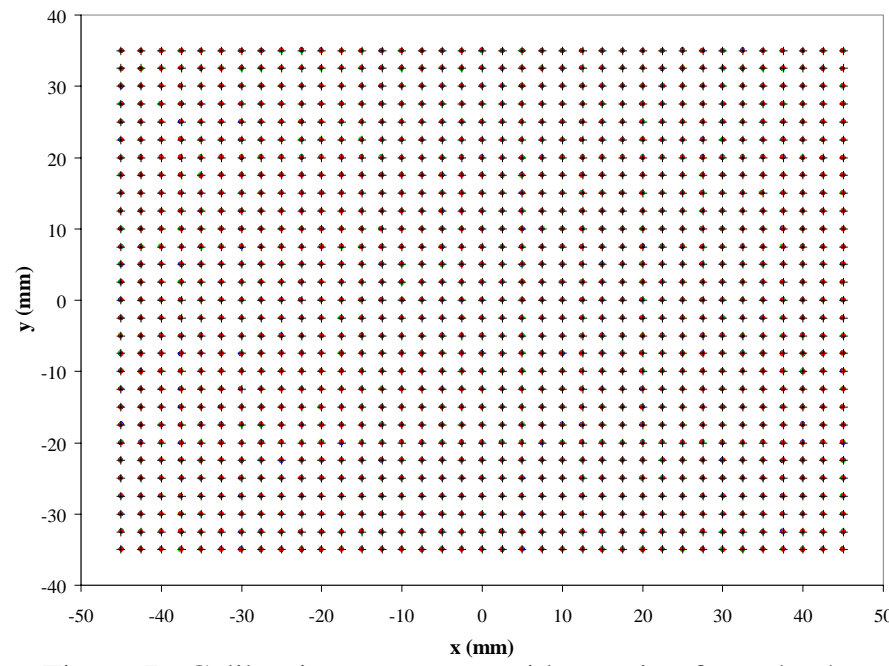

Figure 7: Calibration target centroid mapping from the 4 independent CCD modules to a common reference grid. The rms centroid variation is 0.25 pixels.

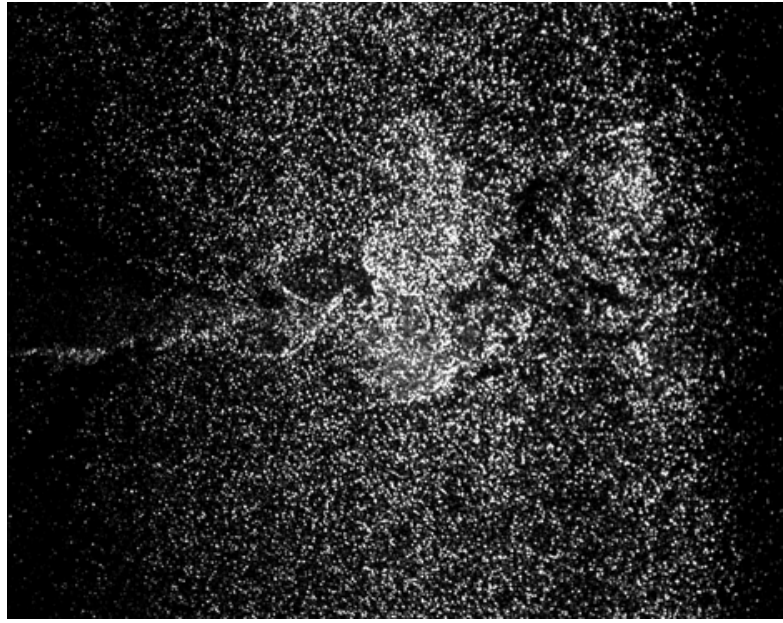

Figure 8: Sample PIV image from high framing rate camera system. a)

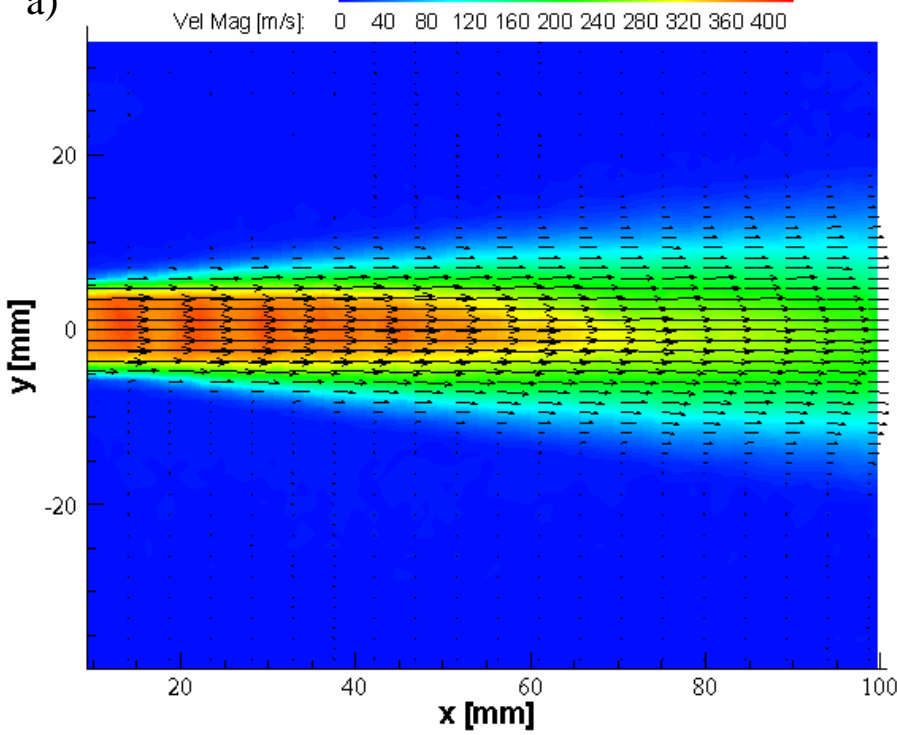

b)

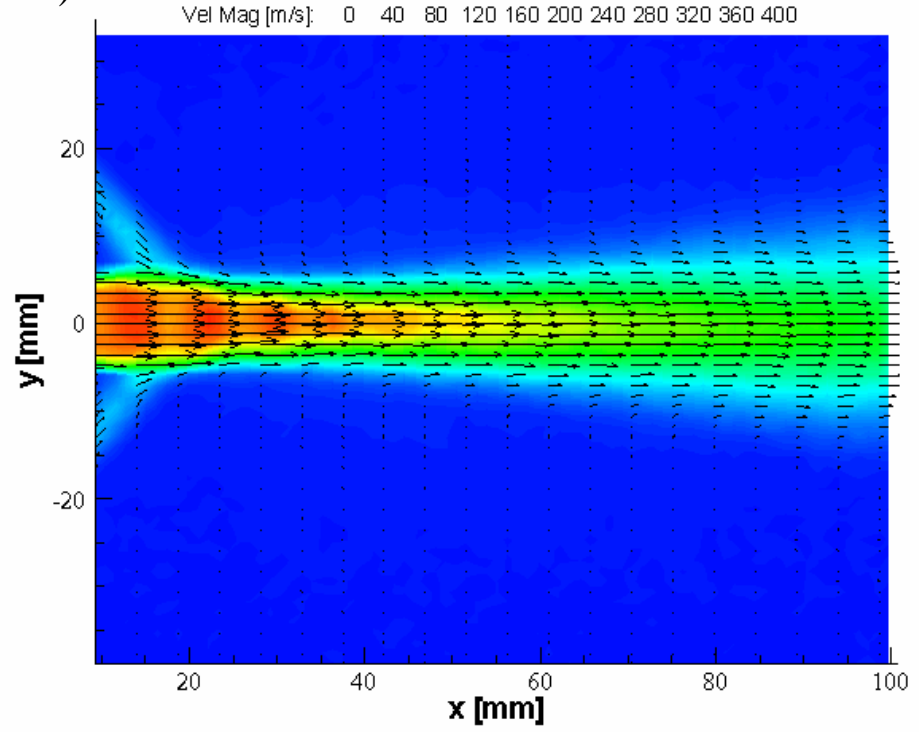

Figure 9: 256 frame ensemble averaged jet flows: a) no-pulse case and b) pulsed jet excitation case. The under expanded jet flow is manifested by the shock diamonds observed in both cases. The effect of the excitation jets is to squeeze down and reduce the length of the jet potential core. 

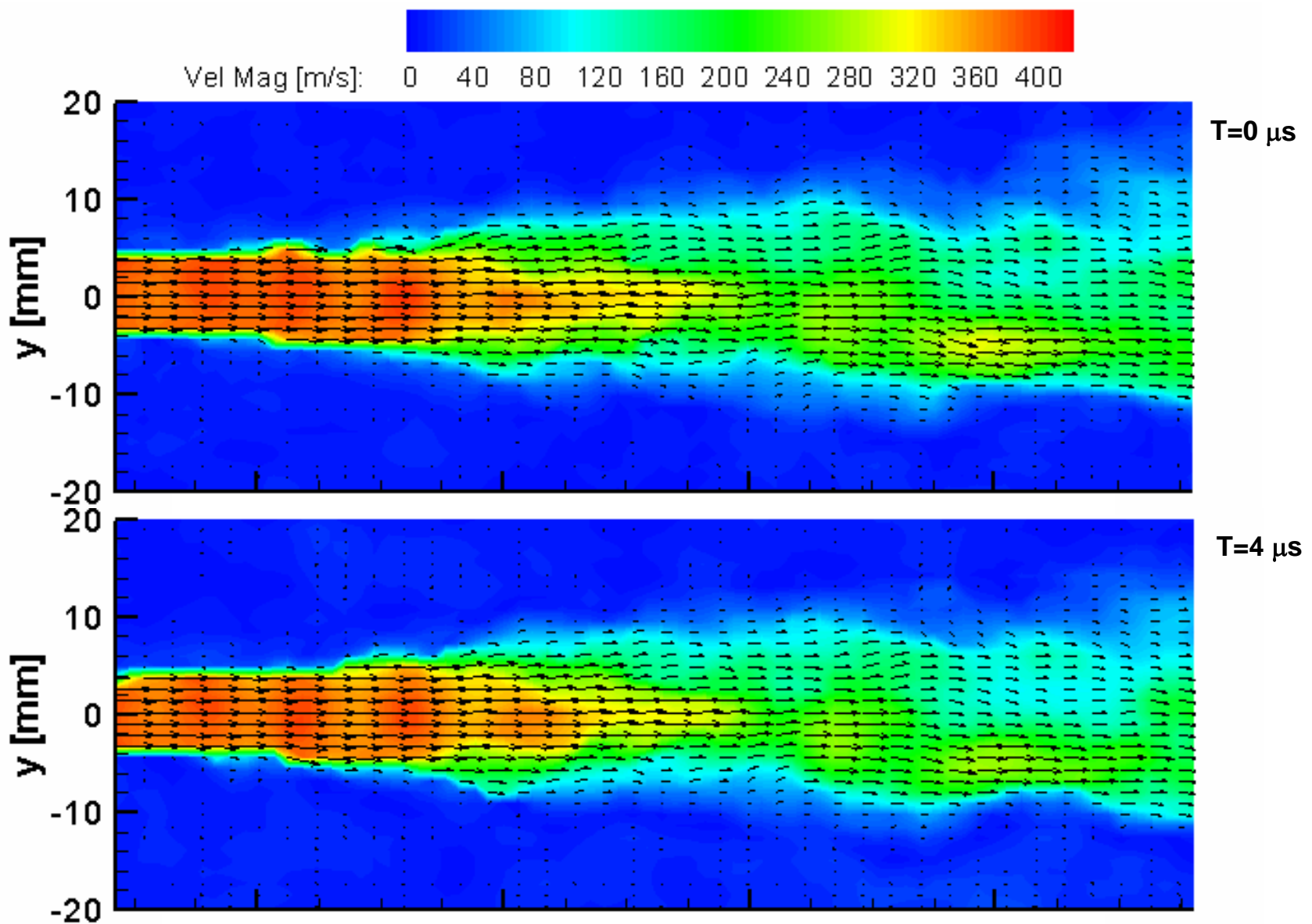

$\mathrm{T}=4 \mu \mathrm{S}$
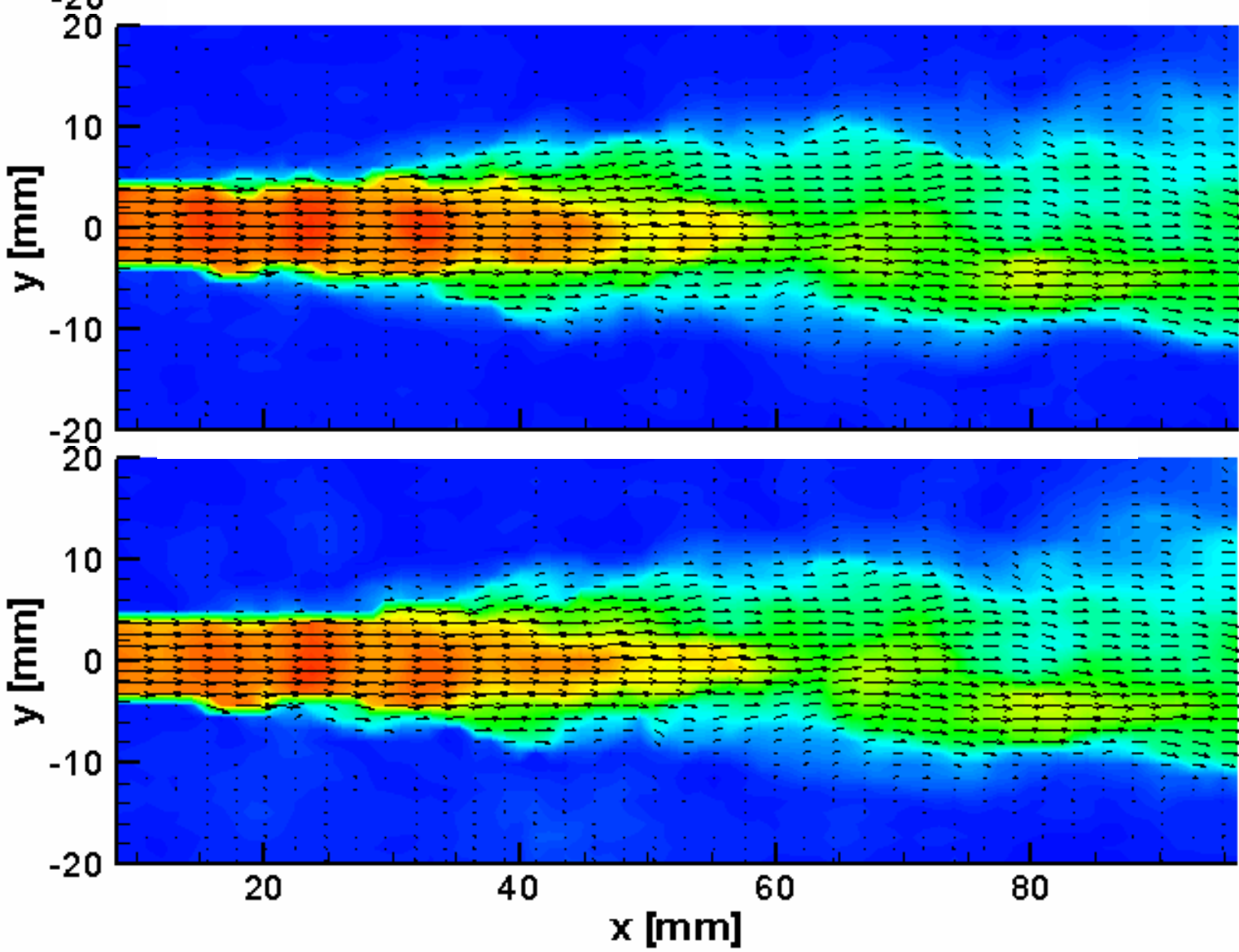

$\mathrm{T}=12 \mu \mathrm{s}$

Figure 10: No-pulse case, where every other map in an equispaced, 7 vector map sequence is shown. The inter-frame time is 2 $\mu \mathrm{s}$ and the time between vector maps is $2 \mu \mathrm{s}$. The flow features are observed moving downstream as the flow evolves in time. 

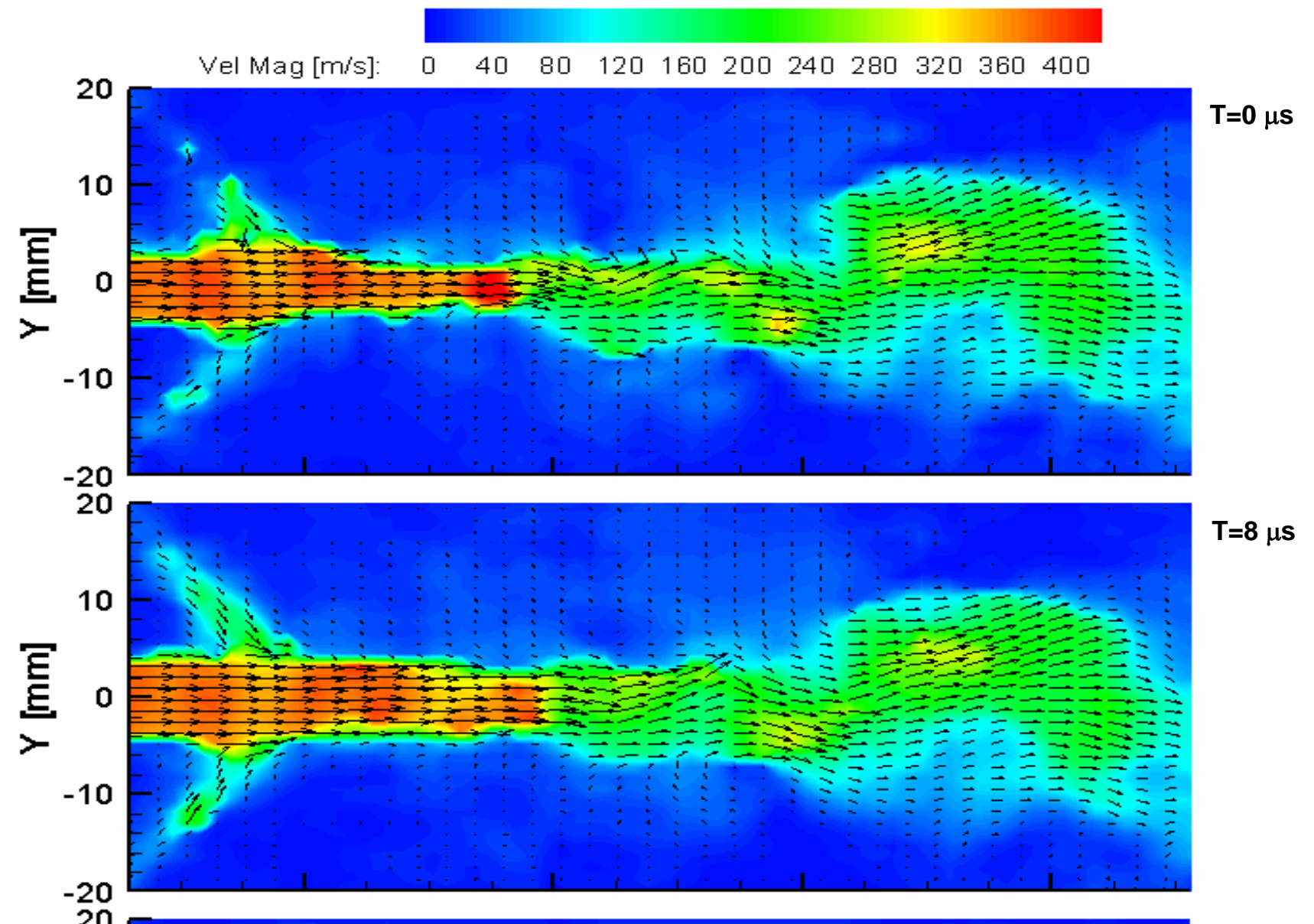

$\mathrm{T}=8 \mu \mathrm{s}$

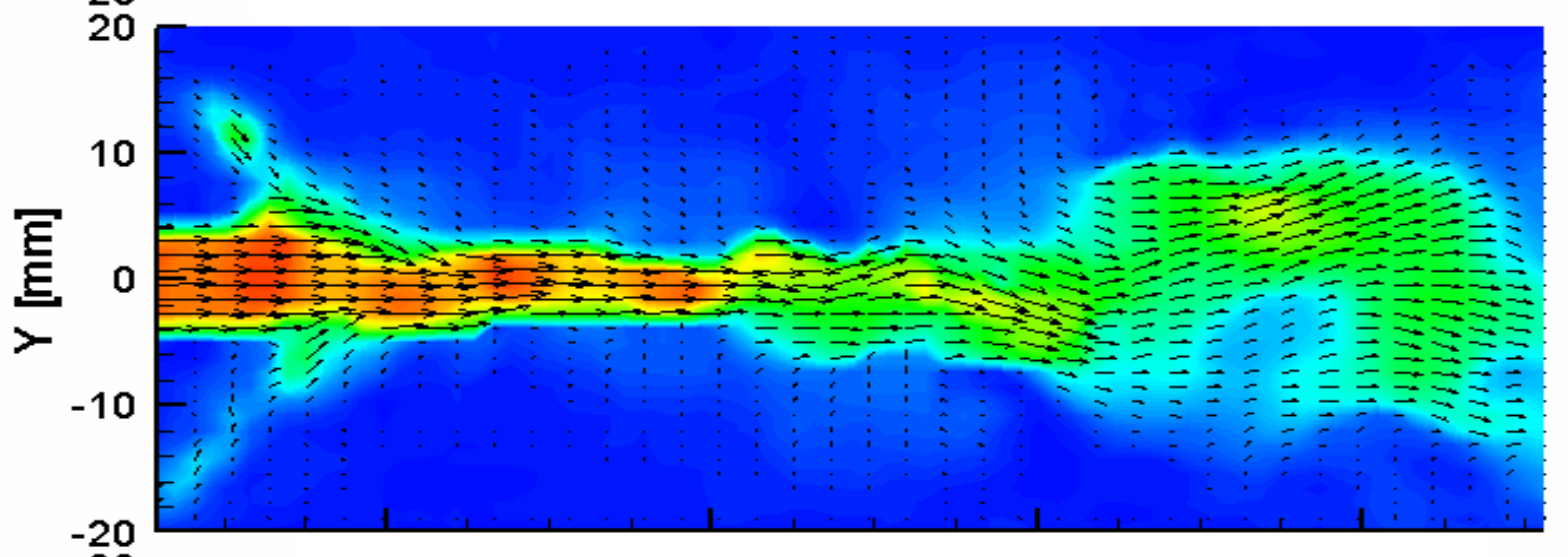

$\mathrm{T}=16 \mu \mathrm{s}$

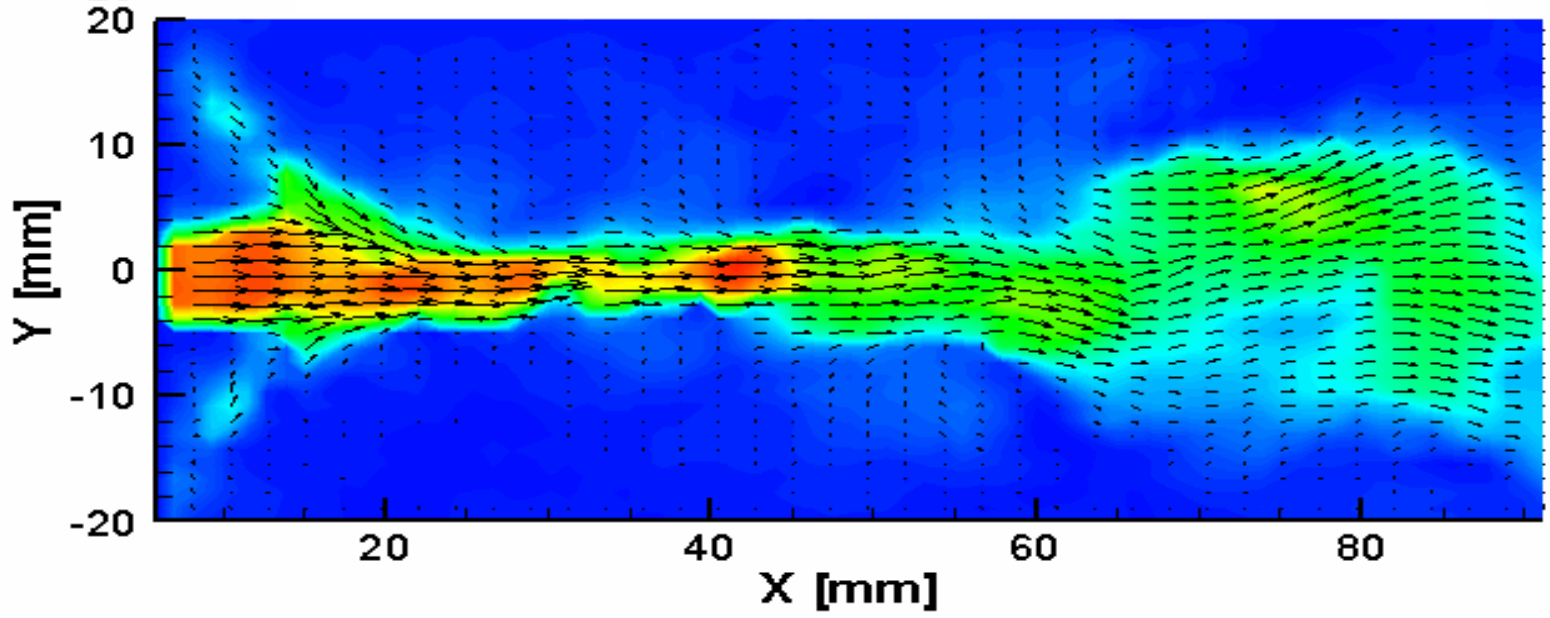

$\mathrm{T}=24 \mu \mathrm{s}$

Figure 11: Four vector map sequence from the pulsed excitation nozzle flow. The inter-frame time is $2 \mu$ s and the time between vector maps is $8 \mu \mathrm{s}$. 